\title{
Family studies on the chromosomal location of the retinoblastoma gene $(\mathrm{Rb}-1)$
}

\author{
J E N MORTEN, D G HARNDEN, AND S BUNDEY \\ From the Department of Cancer Studies, and Department of Clinical Genetics, University of Birmingham, \\ Birmingham
}

SUMMARY The segregation of chromosomes 13 distinguishable by $Q$ band fluorescent polymorphisms has been studied in three families with retinoblastoma. The recombination fraction for two of these families and four families previously reported did not differ significantly from $50 \%$. Since a high recombination fraction has been predicted from chiasma frequency between the centromere of chromosome 13 and $13 \mathrm{q} 14$ these results neither confirm nor refute the location of the autosomal dominant gene predisposing to retinoblastoma in 13q14. The use of fluorescent markers is not suitable for early recognition of gene carriers in families with retinoblastoma.

Predisposition to retinoblastoma can be inherited in an autosomal dominant manner with a penetrance of about $90 \%$. This gene (Rb-1) may be acquired either by germinal mutation or inheritance from a carrier parent. Of patients with retinoblastoma who have inherited the gene, 60 to $70 \%$ are bilaterally affected; on the other hand retinoblastoma patients who do not inherit the gene are invariably unilaterally affected. ${ }^{\mathbf{2}}$

Twenty-six retinoblastoma patients with a constitutional chromosome 13 abnormality have been reported and there are six cases in which the abnormal $\mathrm{D}$ was not identified. With one exception, ${ }^{3}$ all cases where banding was done showed loss of some or all of band 13q14, and by examining banded prophase chromosomes Yunis and Ramsay ${ }^{4}$ were able to detect a sub-band deletion, del $13 q(14 \cdot 1$ : $14 \cdot 3$ ), in a child with unilateral retinoblastoma.

Retinoblastoma patients with a $13 q$ deletion are frequently bilaterally affected and it is possible that $\mathrm{Rb}-1$ is located in 13q14. However, since a deletion may alter the action of genes in both the deleted and uninvolved chromosome regions one must be cautious in assigning the Rb-1 gene to $13 q 14$. Knight et $a l^{5}$ have reported the segregation of $\mathrm{Q}$ banded chromosome 13 variants in four families with retinoblastoma and found a high frequency of discordant segregation of chromosome 13 markers and retinoblastoma.

In this report the segregation of chromosome 13 in three families with retinoblastoma has been studied. Received for publication 5 June 1981

\section{Materials and methods}

Whole venous blood was cultured in Ham's F10 with $10 \%$ bovine serum for 48 hours. Colchicine $(0.002 \%)$ was added one hour before harvesting metaphase cells.

The $\mathrm{Q}$ banding method was a modification of Caspersson et al. $^{6}$ Slides were rehydrated and stained with quinacrine mustard $(50 \mu \mathrm{g} / \mathrm{ml})$ for about 30 minutes, rinsed for 5 minutes in running tap water, and mounted in McIlvaine's buffer, $\mathrm{pH} 7 \cdot 2$.

The description of the markers is according to the International System for Human Cytogenetic Nomenclature (ISCN) 1978. ${ }^{7}$

\section{Patients}

The families were ascertained from index patients with bilateral retinoblastoma located by the Birmingham Regional Cancer Registry. A total of 17 live index patients was ascertained. Four families with more than one case of retinoblastoma were studied and of these three showed useful fluorescent markers of chromosome 13. Family members were studied in their own homes. Information was obtained about other relatives and the eyes of patients, parents, and sibs of patients were examined with an ophthalmoscope after pupil dilation for evidence of regressed tumours.

The pedigree of family 1 (fig 1) is part of a larger pedigree. ${ }^{8}$ The proband in family 1 (III.2) was diagnosed at 2 months of age and treated by left 


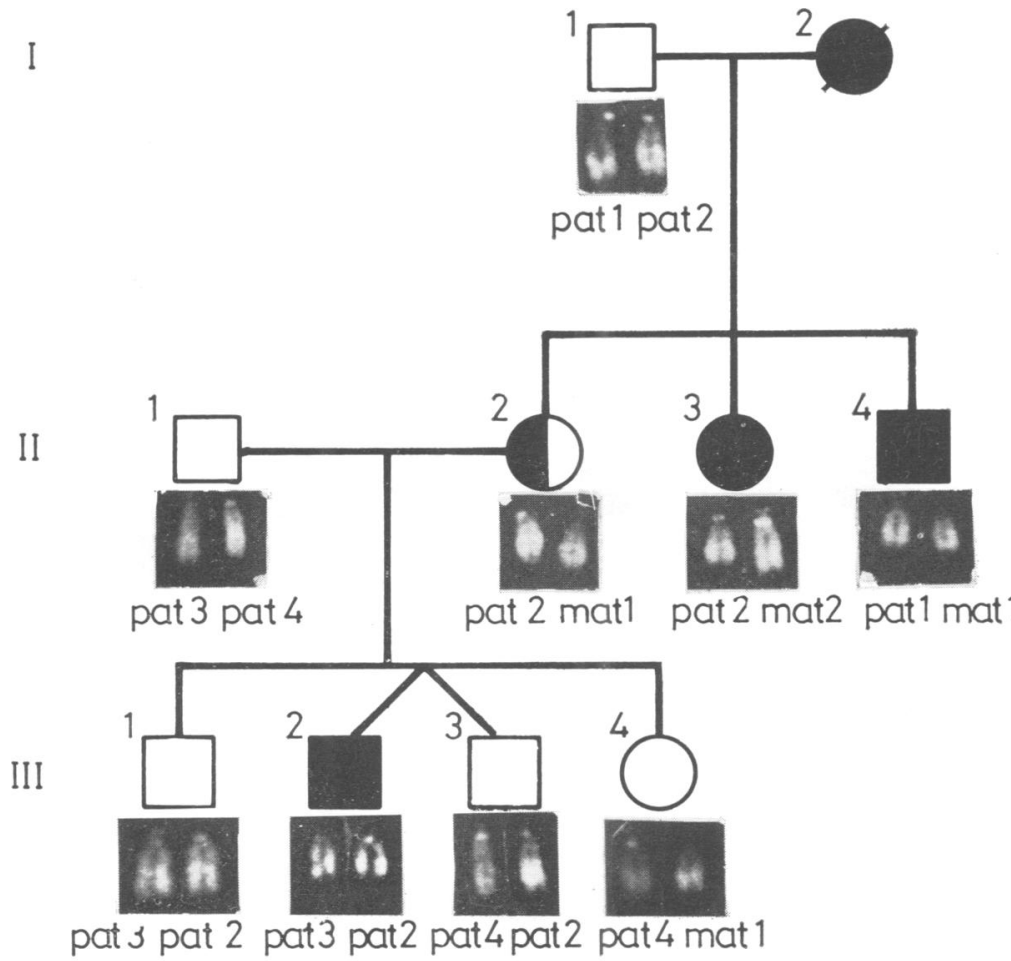

FIG 1 Family 1 showing $Q$ banded chromosomes 13.

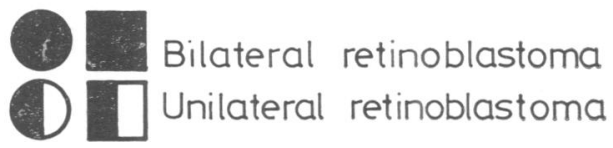

enucleation and irradiation and right irradiation. An osteosarcoma of the left orbit was diagnosed at 7 years of age and he died 6 months later. His mother (II.2) and aunt (II.3) did not have enucleations, but were treated by irradiation as babies. His uncle (II.4) was diagnosed at 7 months of age and was treated with irradiation and bilateral enucleation.

In family 2 (fig 2), the proband (II.3) was diagnosed at 6 months of age and treated by left enucleation; the right eye was treated with radiation starting at 6 months and enucleation was carried out at the age of 2 years. Her father (I.1) had one eye removed as a child in Poland and is assumed to have had unilateral retinoblastoma. In her brother (II.1) bilateral retinoblastomas were recognised at 12 months of age; enucleation of the left eye was performed, but it subsequently became clear that the tumour had spread beyond the orbit. The right eye was treated by irradiation, but the tumour progressed. The boy died of metastases at 9 years of age. Her sister (II.2) has a white lesion in the right retina. This was first thought to be toxoplasmosis, but since

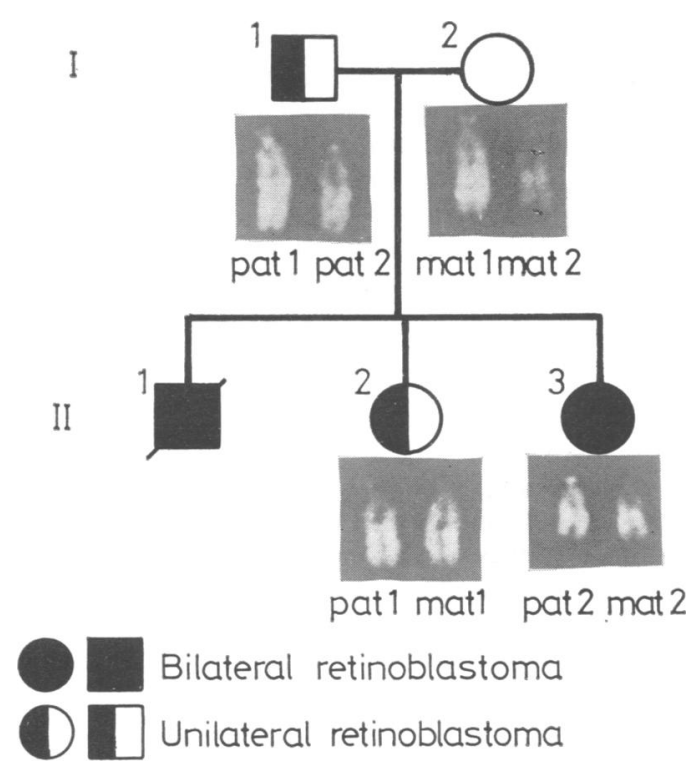

FIG 2 Family 2 showing $Q$ banded chromosomes 13. 


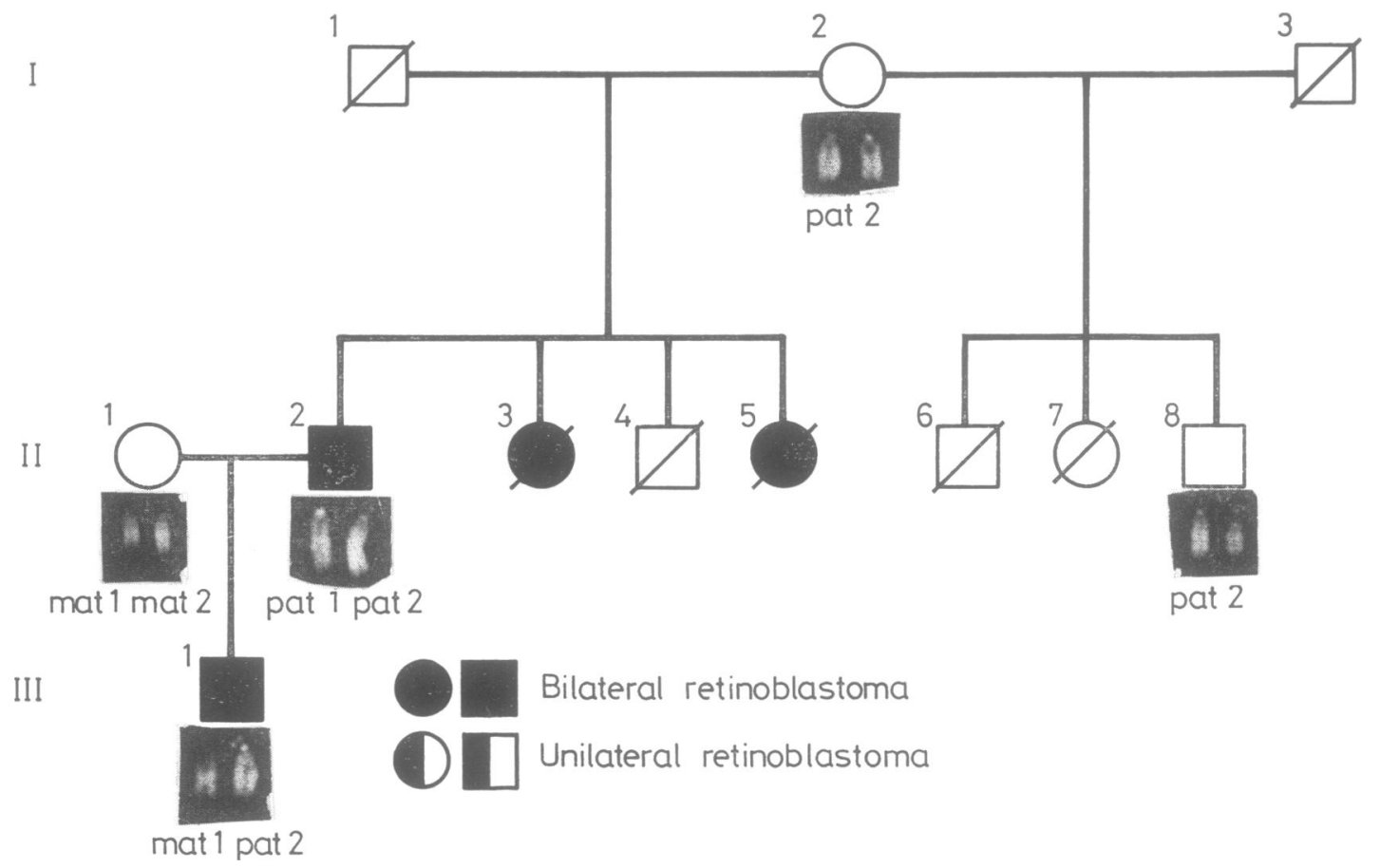

FIG 3 Family 3 showing $Q$ banded chromosomes 13 .

serological tests for toxoplasma were negative and since her risk is 1 in 2 it can be assumed that she carries the retinoblastoma gene. Her left eye is clear.

The proband (III.1) of family 3 (fig 3) was diagnosed at $4 \frac{1}{2}$ months of age and was treated by right enucleation and left irradiation. His father (II.2) developed a squint at 12 months of age and retinoblastoma was diagnosed at 2 years of age and treated by right enucleation and left irradiation. One aunt (II.3) was recognised to have bilateral retinoblastoma at 8 weeks of age and had a left enucleation at 10 weeks; the right eye was enucleated at 5 years and she died a few months later. The other aunt (II.5) had retinoblastoma of the left eye at 4 months and this eye was removed. Tumour developed in the right eye at 11 months and she died during an operation for implantation of radium seeds. Three other sibs died shortly after birth of oesophageal stricture, pneumonia, and Down's syndrome. None was recognised to have retinoblastoma. The paternal grandmother was examined and had no abnormalities of the eyes. The paternal grandfather died at the age of 37 years of tuberculosis.

\section{Results}

FAMILY 1

Fig 1 shows the segregation of the $Q$ banded chromosomes 13 in family 1 . I.1 carries markers 13 pat 1 , which has no centromeric band and has brilliant satellites, and pat 2 , which has an intense band at the centromere but no fluorescent satellites. The retinoblastoma patients II.2, II.3, and II.4 show four distinguishable chromosomes 13 and though the mother with retinoblastoma is dead her markers 13 are clearly mat 1 , which has no fluorescence at the centromere or satellites, and mat 2 , which has an intense centromeric band and pale satellites. Though II.2, II.3, and II.4 all have retinoblastoma they do not have a marker 13 in common. If I. 2 carries the Rb- 1 gene on mat 1 then in generation II there is one recombinant (II.3) out of three sibs. Alternatively if the Rb-1 gene is on mat 2 there are two recombinants (II.2 and II.4) out of three sibs.

II. 2 transmitted mat 1 to one child (III.4) who has not developed retinoblastoma and pat 2 to one child with retinoblastoma. Two children without retinoblastoma inherited pat 2 . In generation III there are two recombinants in four sibs if $R b-1$ is on $13 q$.

FAMILY 2

Fig 2 shows the segregation of the $Q$ banded chromosomes 13 in family 2. 
Retinoblastoma patient I.1 carries markers 13 pat 1 , which has a centromeric band of medium intensity and medium fluorescent satellites, and pat 2 , which has a medium fluorescent centromeric band but no fluorescent satellites. I.2 carries mat 1, which has pale satellites and no centromeric band, and mat 2, which has neither centromeric band nor fluorescent satellites.

II. 2 who is probably an Rb-1 gene carrier has inherited pat 1 from I. 1 and II. 3 has retinoblastoma and has inherited pat 2 from I.1. Since both are probably gene carriers one must be a recombinant for chromosome 13. There is therefore one recombinant in two sibs.

\section{FAMILY 3}

Fig 3 shows the segregation of the $Q$ banded chromosomes 13 in family 3 .

II. 1 carries markers 13 mat 1 , which has neither centromeric band nor satellites, and mat 2 , which has a pale centromeric band and satellites with almost no fluorescence.

II. 2 has retinoblastoma and carries markers 13 pat 1 , which has an intense fluorescent centromeric band and no fluorescent satellites, and pat 2, which has a medium fluorescent centromeric band and medium fluorescent satellites.

III.1 has retinoblastoma and carries markers 13 pat 2 and mat 1.

I. 2 carries marker 13 pat 2 and a chromosome 13 with an intense fluorescent band at the centromere, medium fluorescent satellites, and a larger short arm than pat 2.

Marker pat 1 is clearly inherited from $I .1$ and the sister of I.1 carries two chromosomes 13 identical to pat 1 .

II. 8 carries chromosome 13 pat 2.

Since this family shows multiple affected sibs from unaffected parents either I.1 or I.2 is probably an unaffected $\mathrm{Rb}-1$ gene carrier, but interpretation of this family is complicated by not knowing which of I. 1 or I. 2 is the gene carrier. If I. 1 was the carrier the recombination frequency is $1 / 2$; if $I .2$ carries the $\mathrm{Rb}-1$ gene it is $1 / 3$.

\section{Recombination fraction}

The recombination fraction between retinoblastoma and the fluorescent markers was calculated as a maximum likelihood estimate. ${ }^{9}$ For families 1 and 2 the estimate of the recombination fraction is $50 \%$. If the four families of Knight et al ${ }^{5}$ are included in the calculation the recombination fraction estimate is $38.6 \%$ with a standard error of $15.4 \%$, which is not significantly different from $50 \%$.

\section{Discussion}

None of the three families with retinoblastoma showed a concordant segregation of retinoblastoma and chromosome 13. Knight et $a l^{5}$ found a discordant segregation in three out of four families.

Examples of families like family 3, in which there are multiple affected sibs from unaffected parents, are not uncommon (for example, 60 out of 135 families in Herrmann ${ }^{10}$ ). I.1 or I. 2 could either be an unaffected Rb-1 gene carrier or a gonadal mosaic or, if the delayed mutation hypothesis is correct, a carrier of a premutation. Because of this uncertainty this family was not included in the calculation of the recombination fraction.

A recombination frequency between the centromere and a locus in 13q14 of 27 to $37 \%$ has been estimated from the observed chiasma frequency in normal male meiosis, and in female meiosis the recombination frequency may be nearer $50 \%$. Thus the observed recombination frequency between retinoblastoma and the fluorescent markers of chromosome 13 which is not significantly different from $50 \%$ is compatible with, but neither confirms nor refutes, the location of Rb-1 in 13q14. ${ }^{11}$ Evidence that Rb-1 may not be at $13 \mathrm{q} 14$ has been presented by Matsunaga. ${ }^{12}$

Esterase $D$ has been assigned to $13 q_{14}{ }^{13}$ and Cook et $a l^{14}$ reported a recombination frequency between esterase $\mathrm{D}$ and the centromere of chromosome 13 of $44 \%$ in males and $50 \%$ in females. Studies using esterase $D$ in families with retinoblastoma may be informative as to the location of $\mathrm{Rb}-1$ in 13q14.

The high recombination frequency between retinoblastoma and the chromosome 13 fluorescent markers makes the use of fluorescent markers unsuitable for early recognition of Rb-1 gene carriers in families with retinoblastoma.

We would like to thank $\mathrm{Dr} G$ Gale for the calculation of the recombination fractions; $\mathrm{Dr}$ J A H Waterhouse of the Birmingham Regional Cancer Registry for locating bilateral retinoblastoma patients; those consultant ophthalmologists who kindly allowed us to study their patients; Susan Williams for help in preparing photographs; the Cancer Research Campaign for the grant which supported this work.

\section{References}

1 Vogel F. Genetics of retinoblastoma. Hum Genet 1979; 52:1-54.

2 Matsunaga E. Hereditary retinoblastoma: delayed mutation v host resistance. Am J Hum Genet 1978;30: 406-24. 
3 Davison EV, Gibbons B, Aherne GES, Roberts DF. Chromosomes in retinoblastoma patients. Clin Genet 1979;15:505-8.

4 Yunis JJ, Ramsay N. Retinoblastoma and sub-band deletion of chromosome 13. Am J Dis Child 1978;132: 161-3.

5 Knight LA, Gardner A, Gallie BL. Familial retinoblastoma: segregation of chromosome 13 in four families. Am J Hum Genet 1980;32:194-201.

- Caspersson T, Lomakka G, Zech L. The 24 fluorescence patterns of the human metaphase chromosomes distinguishing characters and variability. Hereditas 1971 ;67: 89-102.

7 ISCN. An international system for human cytogenetic nomenclature. Cytogenet Cell Genet 1978;21:309-404.

8 Morten JEN. A study of retinoblastoma as an example of genetic predisposition to cancer in man. PhD thesis, University of Birmingham, 1980.

9 Cavalli-Sforza LL, Bodmer WF. The genetics of human populations. San Francisco: Freeman, 1971.

10 Herrmann J. Delayed mutation model: carotid body tumours and retinoblastoma. In: Mulvihill JJ, Miller
RW, Fraumeni JF, eds. Genetics of human cancer. New York: Raven Press, 1977:417-38.

11 Palmer RW, Hultén MA. Chiasma derived genetic maps and recombination fractions: chromosome 13 with $\stackrel{\vec{f}}{+}$ reference to the proposed 13q14 retinoblastoma locus. J Med Genet 1982;19:125-9.

12 Matsunaga E. Retinoblastoma: host resistance and 13qchromosomal deletion. Hum Genet 1980;56:53-8.

13 Sparkes RS, Sparkes MC, Wilson MG, et al. Regional assignment of genes for human esterase $D$ and retinoblastoma to chromosome band 13q14. Science 1980;208: 1042-4.

14 Cook, PJL, Robson EB, Rogers PA, Noades JE, Buckton KE, Watson AR. Family studies on nucleoside phosphorylase and the short arm of chromosome 14. Ann Hum Genet 1981 ;45:253-60.

Requests for reprints to Dr J E N Morten, MRC Clinical and Population Cytogenetics Unit, Western General Hospital, Edinburgh EH4 2XU. 